\title{
Chronic transmural atrial ablation by using bipolar radiofrequency energy on the beating heart
}

Sunil M. Prasad, MD

Hersh S. Maniar, MD

Richard B. Schuessler, PhD

Ralph J. Damiano, Jr, MD

From the Division of Cardiothoracic Surgery, Washington University School of Medicine, St Louis Mo.

Supported by a Research Grant from AtriCure Inc and by National Institutes of Health grants 5 R01 HL32257 and T32 HL07275.

Received for publication Oct 31, 2001; revisions requested Feb 5, 2002; revisions received March 7, 2002; accepted for publication March 24, 2002.

Address for reprints: Ralph J. Damiano, Jr, MD, 1 Barnes Jewish Plaza, Division of Cardiothoracic Surgery, St Louis MO 63110 (E-mail: damianor@msnotes.wustl.edu).

J Thorac Cardiovasc Surg 2002;124:708-13

Copyright (C) 2002 by The American Association for Thoracic Surgery

$0022-5223 / 2002 \$ 35.00+0 \quad \mathbf{1 2 / 1 / 1 2 5 0 5 7}$

doi:10.1067/mtc.2002.125057
Background: Recently, there has been renewed interest in the development of minimally invasive procedures to treat atrial fibrillation. Unipolar radiofrequency catheters are plagued by poor results, in part because of their inability to produce transmural lesions. This study tested the ability of bipolar radiofrequency energy to create chronic transmural lesions on the beating heart that isolated atrial myocardium.

Methods: Five sheep underwent a right thoracotomy. Baseline pacing was performed from the following targeted areas: right atrial appendage, superior vena cava, inferior vena cava, and right pulmonary veins. A cuff of atrial myocardium around the targeted tissue was clamped between the 2 arms of the device. Radiofrequency energy was delivered at $750 \mathrm{~mA}$ and continued until the tissue conductance between the electrodes reached a stable minimum level. After ablation, pacing was used to document tissue isolation. The animals survived for 30 days.

Results: Twenty circumferential lesions were produced at the initial operation. The mean ablation time was $9.3 \pm 4.0$ seconds, and the mean peak temperature was $48.4^{\circ} \mathrm{C} \pm 6.4^{\circ} \mathrm{C}$. All lesions acutely and chronically isolated the targeted tissue. Trichrome staining showed that all lesions were transmural. There were no instances of pulmonary vein stenosis or thrombosis.

Conclusions: Bipolar radiofrequency energy can produce permanent transmural linear lesions on the beating heart. Online measurement of tissue conductance reliably predicted lesion transmurality. This new technology may enable surgeons to perform a curative minimally invasive operation for atrial fibrillation on the beating heart.

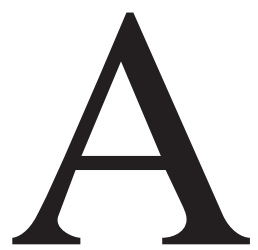

trial fibrillation is the most common dysrrhythmia in the United States, with an incidence of $2 \%$ in the general population. ${ }^{1}$ In the elderly the incidence can be as high as $6 \%$. Even with the aggressive treatment of atrial fibrillation, there still exists a significant mortality and morbidity from this disease. Patients are at an increased risk for stroke and other thromboembolic disease..$^{2,3}$ They also have increased morbidity as a result of atrioventricular dysynchrony and compromised hemodynamics. It is estimated that atrial fibrillation accounts for 1.4 million outpatient visits and 227,000 hospitalizations each year in the United States alone. ${ }^{4}$ The current medical regimen consists of anticoagulation, as well pharmacologic strategies designed both to control heart rate and to convert the patient to normal sinus rhythm. This regimen, although effective in treating the symptoms of the disease, is by no means a cure. The patient is left with the significant morbidity of anticoagulation and the side effects of the antiarrhythmic drugs. Moreover, rate control alone does not address the impaired hemodynamics or thromboembolic risk.

The development of the Cox maze procedure has offered a cure for atrial 
fibrillation. ${ }^{5}$ This surgical approach has achieved success rates of greater than $90 \%$, and it has been established as the gold standard against which other interventions are judged. The Cox maze procedure is based on the theory that atrial fibrillation is the result of macroreentrant circuits. ${ }^{6}$ By abolishing these circuits with lines of conduction block created by means of carefully placed surgical incisions, atrial fibrillation can be eliminated. Despite the clinical success of the maze procedure, it has not gained widespread acceptance because of its invasiveness and the length of time required to create the complicated set of lesions. Recently, interest has turned toward the development of a less invasive procedure. Ideally, this procedure would require less operative time, could be performed through a small incision or endoscopically, and would not require a prolonged period of cardiopulmonary bypass and cardioplegic arrest.

Over the past 10 years, there have been significant advances in the development of radiofrequency (RF) energy as a means to ablate myocardial tissue. ${ }^{7}$ This energy, delivered in an epicardial or endocardial manner, is capable of producing myocardial necrosis and has the ability to electrically isolate myocardial tissue. RF energy has been used by electrophysiologists in the ablation of accessory pathways and atrioventricular node reentry with significant success. ${ }^{8}$ There also has been much interest in RF ablation for the treatment of atrial fibrillation. However, difficulty remains in use of catheter-based techniques to create reproducible transmural lesions. These procedures have required lengthy fluoroscopic exposure times and have been plagued both by a high incidence of complications and low success rates.9,10 The lack of success of catheter-based techniques has led to a resurgence of interest in surgical procedures to treat atrial fibrillation. Unfortunately, unipolar RF energy cannot reliably create transmural ablation on the beating heart. ${ }^{11}$ Our goal was to investigate whether RF energy delivered by means of a bipolar device could reproducibly create lesions in a rapid and controlled manner that was capable of electrically isolating myocardial tissue.

\section{Methods}

Five adult sheep weighing 35 to $45 \mathrm{~kg}$ were used in this study. The animals were initially anesthetized with intramuscular ketamine (30 mg/kg), followed by inhaled 3\% isoflurane. Arterial and venous access were achieved through a cut down of the carotid artery and external jugular vein, respectively. The arterial pressure and electrocardiographic results were monitored continuously. A 7-cm right anterolateral thoracotomy was made in the fourth intercostal space. The pericardium was opened, and the right atrium and both vena cavae were exposed. The inferior vena cava (IVC), superior vena cava (SVC), and right pulmonary veins (RPVs) were circumferentially dissected and isolated with umbilical tape. The lesion set in this study was designed to maximize the potential circumferential lesion site to evaluate electrical isolation by pacing studies and does not represent a variation of the Cox maze procedure.
The heart was paced from the right atrial body and appendage, and pacing thresholds were recorded. Pacing was also performed from the right-sided pulmonary veins, as well as from both vena cavae, at a distance of $1 \mathrm{~cm}$ from the atriocaval junction. The animal was given intravenous heparin $(200 \mathrm{U} / \mathrm{kg})$, and an activated clotting time of greater than 200 seconds was maintained throughout the study.

A bipolar RF ablation device was used to create the lesions (Atricure Inc, Cincinnati, Ohio). The RF generator is able to control both voltage and current delivery to the electrodes. A laptop computer with Labview version 5.1 (National Instruments, Austin, Tex) was used to monitor and record temperature, time, current, voltage, impedance, conductance, and energy. Temperature was recorded $1 \mathrm{~mm}$ from the electrode edge. The computer system displayed these variables in a real-time continuous graphic format.

Atrial lesions were created by clamping target tissue between the 2 electrode arms of the device. A cuff of atrial myocardium was ablated around the right atrial appendage (RAA), SVC, IVC, and right-sided pulmonary veins. RF energy was delivered at $75 \mathrm{~V}$ and $750 \mathrm{~mA}$. The ablation was continued until the tissue conductance between the 2 electrodes decreased and achieved a steady state for 2 seconds (Figure 1). Tissue conductance was chosen as an indicator of a complete transmural ablation. Electrical isolation was defined as the inability to capture the body of the right atrium by pacing inside the isolated segment with a stimulus strength of $20 \mathrm{~mA}$. The animals survived for 30 days after ablation. The animals received $325 \mathrm{mg}$ of aspirin each morning after the operation.

On day 30, a median sternotomy was performed, and the heart was exposed. Pacing at $10 \mathrm{~mA}$ was used to document chronic electrical isolation. The animal was put to death with sodium pentobarbital, and the heart was excised en bloc. The atrium and pulmonary veins were examined for any evidence of thrombus or stricture. The RF lesions were grossly examined, and tissue was stained with Masson Trichrome to assess transmurality.

All animals received humane care in compliance with the "Guide for the Care and Use of Laboratory Animals" published by the National Institutes of Health (National Institutes of Health publication no. 85-23, revived 1985).

\section{Results}

\section{Operative Results}

In one animal it was not possible to dissect circumferentially around the IVC or RPVs because of adhesions present at the initial operation. There were no intraoperative complications or postoperative morbidity or mortality in this series. The animals were continuously monitored in the immediate postoperative period (48 hours) and remained in normal sinus rhythm. Preoperatively and at 30 days, all the animals were in normal sinus rhythm.

\section{RF Lesion Delivery}

A total of 20 circumferential lesions were performed on cuffs of atrial myocardium surrounding the SVC, IVC, and RPVs and on the RAA (Table 1). In the last 2 animals, a second RAA lesion was created to connect the initial RAA 


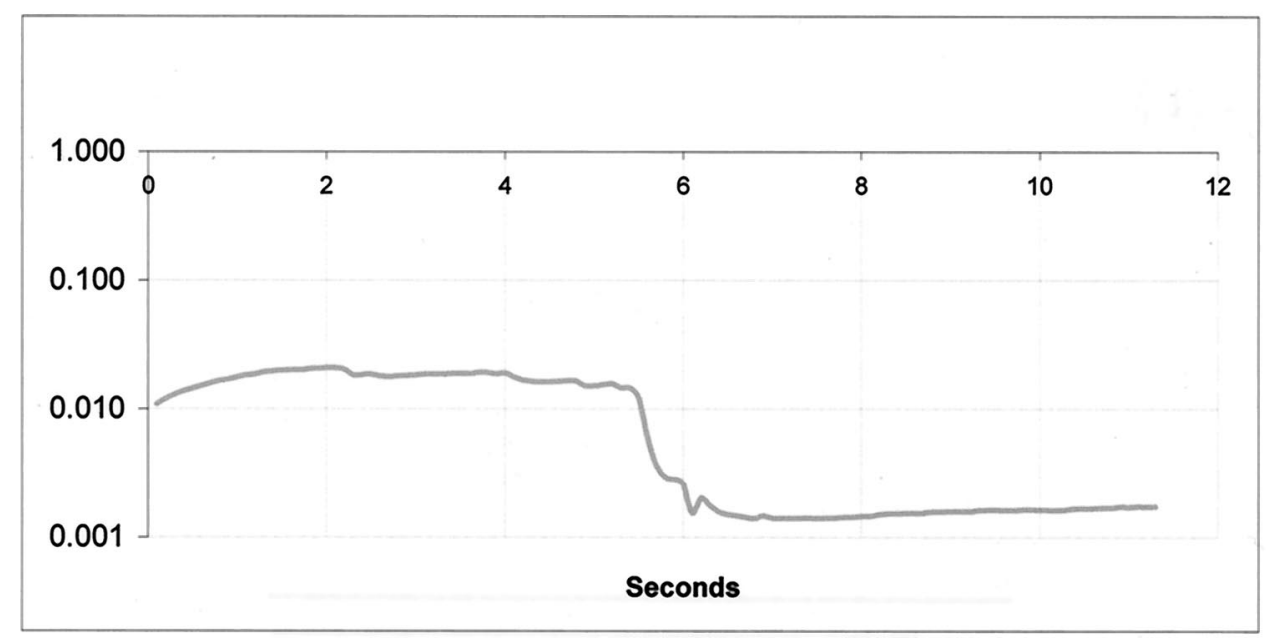

Figure 1. A real-time graph of tissue conductance (measured in Mhos on the $Y$ axis) between the 2 electrodes on the arms of the device and time (measured in seconds on the $X$ axis). This graph was taken from an RAA lesion on the beating heart. It shows the decrease in tissue conductance at 5.5 seconds and a stable minimal level afterward. Energy was delivered for 11 total seconds during this ablation.

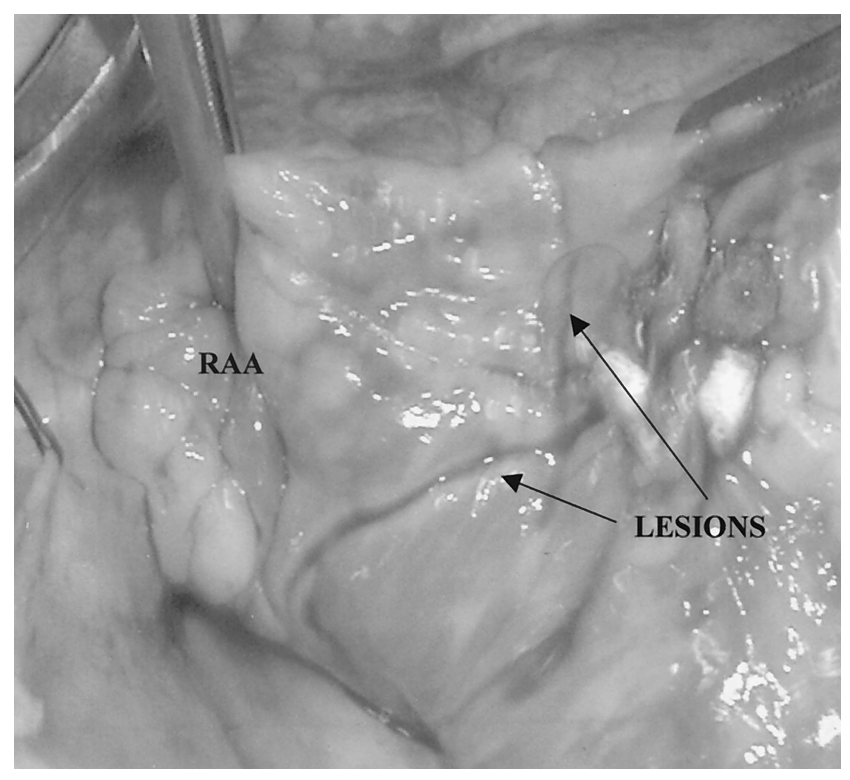

Figure 2. An in vivo picture of 2 connecting lesions of the body of the right atrium. These 2 lines of block electrically isolated the appendage from the body of the right atrium.

lesion and to produce an isolated section of myocardium. In both animals the lesion lines overlapped smoothly, and the enclosed myocardium was isolated (Figure 2).

The mean ablation time was $9.3 \pm 4.0$ seconds (range, 6-23.2 seconds), with a mean peak temperature of $48.3^{\circ} \mathrm{C} \pm$ $6.4^{\circ} \mathrm{C}$. For lesions $(n=7)$ created on the RAA, the mean ablation time was $9.4 \pm 3.4$ seconds (range, 6.5-14.8 seconds), and the mean peak temperature was $47.1^{\circ} \mathrm{C} \pm 6.9^{\circ} \mathrm{C}$.
TABLE 1. Ablation summary

\begin{tabular}{lrrccc}
\hline $\begin{array}{l}\text { Lesion } \\
\text { site }\end{array}$ & No. & \multicolumn{1}{c}{ Time (s) } & $\begin{array}{c}\text { Temperature } \\
\left({ }^{\circ} \mathbf{C}\right)\end{array}$ & $\begin{array}{c}\text { Acute } \\
\text { isolation }\end{array}$ & $\begin{array}{c}\text { Chronic } \\
\text { isolation }\end{array}$ \\
\hline RAA & 7 & $9.4 \pm 3.4$ & $47.1 \pm 6.9$ & $100 \%$ & $100 \%$ \\
SVC & 5 & $11.68 \pm 6.6$ & $51.7 \pm 8.6$ & $100 \%$ & $100 \%$ \\
IVC & 4 & $7.4 \pm 0.9$ & $46.7 \pm 2.5$ & $100 \%$ & $100 \%$ \\
RPV & 4 & $7.7 \pm 1.3$ & $48.1 \pm 5.9$ & $100 \%$ & $100 \%$ \\
\hline
\end{tabular}

The wall thickness of the RAA ranged from 2 to $2.5 \mathrm{~mm}$. The average energy delivered was $168.6 \pm 68.9 \mathrm{~J}$.

For lesions $(n=5)$ created on the SVC, the mean ablation time was $11.7 \pm 6.6$ seconds (range, 7-23.2 seconds), with a mean peak temperature of $51.7^{\circ} \mathrm{C} \pm 8.6^{\circ} \mathrm{C}$. The wall thickness of the SVC ranged from 0.5 to $0.8 \mathrm{~mm}$. The average energy delivered was $267.6 \pm 257.2 \mathrm{~J}$.

For lesions $(\mathrm{n}=4)$ created on the IVC, the mean ablation time was $7.4 \pm 0.9$ seconds (range, 6.3-8.2 seconds), with a mean peak temperature of $46.7^{\circ} \mathrm{C} \pm 2.5^{\circ} \mathrm{C}$. The wall thickness of the IVC ranged from 0.4 to $0.8 \mathrm{~mm}$. The average energy delivered was $101.0 \pm 16.43 \mathrm{~J}$.

For lesions $(n=4)$ created on the RPVs, the mean ablation time was $7.7 \pm 1.3$ seconds (range, 6.0-9.0 seconds), with a mean peak temperature of $48.1^{\circ} \pm 5.9^{\circ} \mathrm{C}$. The wall thickness of the RPVs varied depending on the fat pad surrounding the RPV-right atrium junction (range, 1.1-5 $\mathrm{mm})$. The average energy delivered was $102.3 \pm 38.5 \mathrm{~J}$.

\section{Pacing Results}

Before ablation, pacing was performed from all potential target tissues sites (20/20) $1 \mathrm{~cm}$ distal to the junction with the atrial myocardium. Pacing thresholds were less than or 


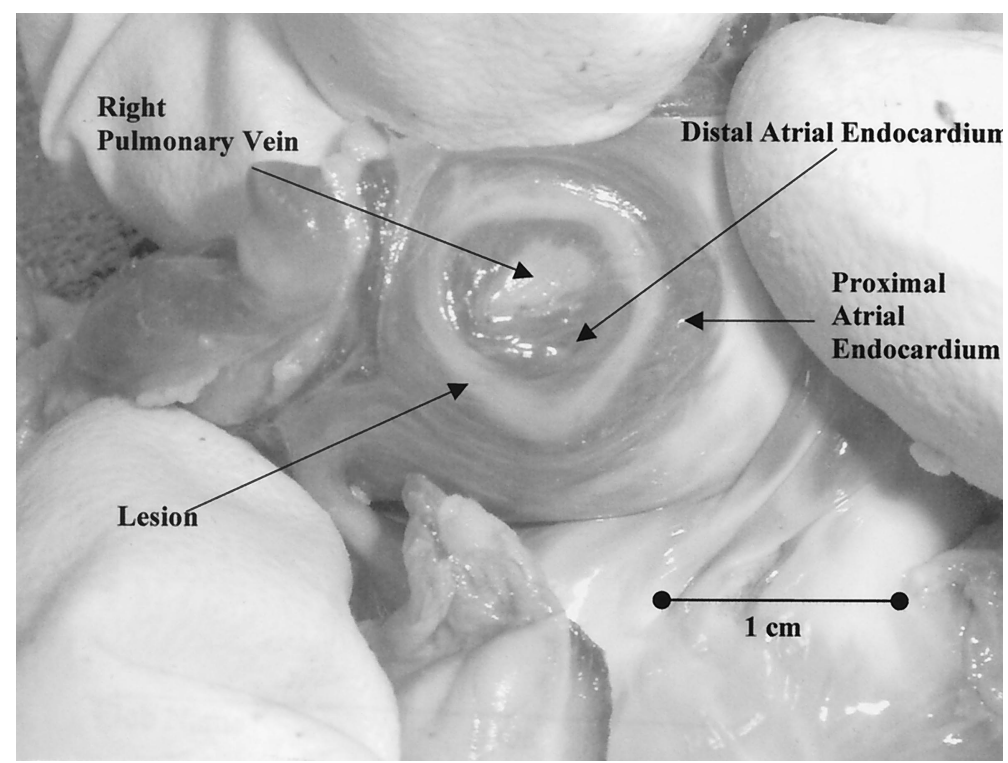

Figure 3. An ex vivo picture of the superior RPV. The device is able to isolate a cuff of atrial tissue at the RPV-left atrial junction by producing a continuous linear lesion circumferentially around the targeted tissue. There was no gross pulmonary vein stenosis or thrombosis.

equal to $1.5 \mathrm{~mA}$ at every site. After ablation, pacing stimuli from all sites were unable to capture the body of the right atrium at stimulus strengths of up to $20 \mathrm{~mA}$. Thus electrical isolation was documented for all 20 ablation lesions. After 30 days, pacing studies documented continued isolation in all 20 lesions.

\section{Histology}

Examination of the atria, vena cavae, and pulmonary veins revealed no evidence of thrombus or stricture formation. All lesions were examined postmortem by using Masson Trichrome staining. Histology demonstrated replacement of atrial myocardium at the ablation site with fibrin and collagen matrix. All lesions were transmural, continuous, and linear (Figures 3 and 4). The lesion width was discrete and varied depending on the depth and time of the lesion. In areas of thin walls (ie, IVC and SVC), the lesion width was fairly constant at $1 \mathrm{~mm}$. On thicker areas of the atrium (ie, the appendage), where the wall thickness was up to $5 \mathrm{~mm}$, the lesion width was increased but never greater than $2 \mathrm{~mm}$.

An interesting finding in this study was that despite creating a transmural lesion that completely isolated electrical activity in the RAA, the appendage remained viable, with no evidence of necrosis (Figure 5). This indicates that although bipolar RF energy causes transmural necrosis of myocardial tissue, it might preserve the microvasculature, at least to the RAA. Further studies are needed to investigate this hypothesis.

\section{Discussion}

Atrial fibrillation is a major source of morbidity, particularly in the elderly population. ${ }^{3}$ Despite advances in transvenous endocardial catheter-based techniques, there is still no consistent cure for atrial fibrillation, except the surgical Cox maze procedure. The Cox maze procedure creates transmural lesions in the myocardium with a scalpel that forms the lines of conduction block, terminating the reentrant circuits that cause AF. Unfortunately, alternative epicardial and endocardial energy sources have failed to produce transmural lesions in a consistent fashion. This might be one of the reasons for the lower success rates seen after catheter-based procedures. ${ }^{12,13}$

Unipolar RF catheters require ablation times measured in minutes, and multiple applications are often required to produce a transmural lesion. These catheters produce electrode temperatures of between $80^{\circ} \mathrm{C}$ and $90^{\circ} \mathrm{C}$ and can cause severe thermal injury to adjacent tissue. The biggest limitation of epicardial unipolar RF ablation is that it is nearly impossible to make a transmural lesion on the beating heart because of the intracavitary heat sink caused by the circulating blood volume. This shortcoming is compounded by the fact that physicians have no way of determining the depth or transmurality of the lesion with presently available unipolar RF endocardial and epicardial catheters. A final challenge of ablation with unipolar catheters is the need to maintain constant approximation to the tissue. The physics of RF energy ablation are based on the theory that the energy source and the tissue must be in intimate contact. ${ }^{12}$ 


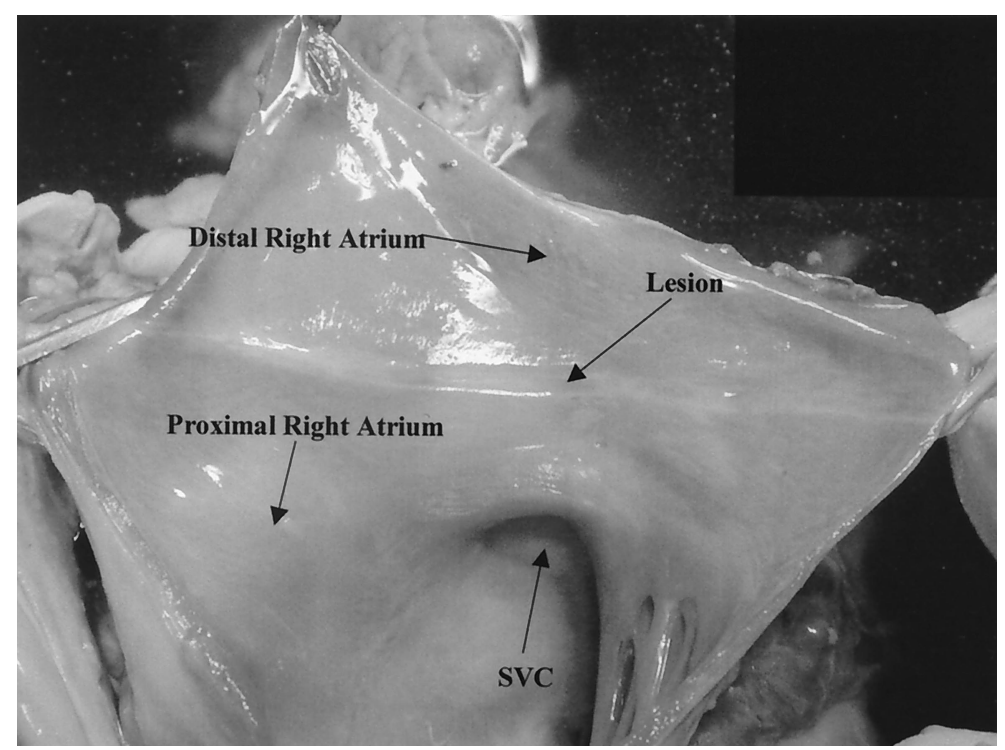

Figure 4. An ex vivo picture of a lesion at the junction of the SVC and the body of the right atrium after 30 days. The anterior portion of the right atrium was opened so that the lesion could be better visualized. The lesion was continuous, linear, and discrete.

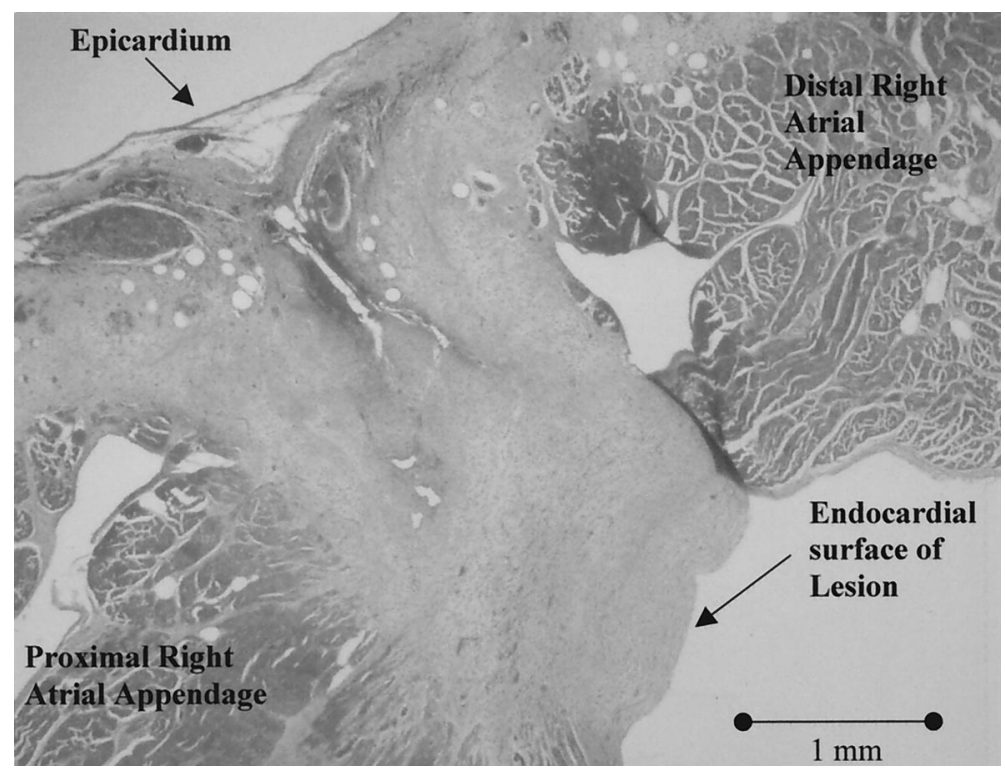

Figure 5. A lesion on the anterior surface of the RAA stained with Masson Trichrome stain. (Original magnification $10 \times$.) In every instance the RAA tip was viable, and the lesion was transmural. In this ablation the width of the lesion was $1.3 \mathrm{~cm}$, and the depth was $2.5 \mathrm{~cm}$.

Uneven pressure or a momentary lapse of contact increases the energy required and results in nonuniformity of the lesion.

There is a pressing clinical need for a device that can reproducibly and reliably perform full-thickness endocardial and epicardial ablation. The bipolar RF device used in this study was able to meet these clinical needs. The catheter was able to consistently produce transmural linear lesions. The time required to produce a transmural linear lesion of up to $5 \mathrm{~cm}$ in length averaged only 9 seconds. By shortening the time required to produce a functional lesion, the tissue was exposed to less energy and less heat. The surface temperature of the electrode was maintained at more physiologic levels $\left(40^{\circ} \mathrm{C}-60^{\circ} \mathrm{C}\right)$, and peak temperature $1 \mathrm{~mm}$ 
from the electrode was significantly lower than the $80^{\circ} \mathrm{C}$ to $90^{\circ} \mathrm{C}$ achieved with unipolar RF catheters.

Histologic evaluation documented that the lesions were discrete, with minimal damage of the surrounding tissue. Average lesion width was less than $2 \mathrm{~mm}$. This is even less damage then seen with a traditional surgical incision. Hypothetically, this might result in a better preservation of atrial transport function, one of the critical outcome measurements after the Cox maze procedure. The lower temperatures and more discrete lesions should decrease the risk of both intraoperative and postoperative thromboembolic complications or stricture. No intracavitary thrombi were found in this chronic study. Moreover, there was also no evidence of pulmonary vein stenosis. This complication has been reported to be as high as 39\% acutely and $8 \%$ chronically after unipolar RF ablation. ${ }^{14,15}$ This is further testimony to the lack of collateral tissue damage with this technique.

The computer system provided with this bipolar RF device monitored multiple variables in a graphic format. Temperature, current, impedance, conductance, voltage, energy, and time are continuously displayed in real time. This provides the surgeon with instantaneous feedback as to the progress of the lesion during ablation. Changes in tissue conductance provided a $100 \%$ correlation with lesion transmurality in this study. As the lesion progressed through the myocardium, the tissue conductance decreased between the electrodes. Once the lesion became transmural, the tissue conductance reached a plateau and did not change. This is the first reported device to have an online measurement of transmurality. This has the advantage of allowing the surgeon to stop ablation as soon as the lesion is completed, protecting the atrial myocardium from unnecessary energy, excessive necrosis, and scarring.

This chronic survival study has demonstrated, for the first time, an efficacious method to create transmural atrial lesions from the epicardium on the beating heart. This technology has the potential to treat atrial fibrillation in a definitive, safe, and expeditious manner. A beating-heart maze procedure would eliminate the morbidity of placing patients on cardiopulmonary bypass and crossclamping the aorta and at the same time offer a more reliable cure of this arrhythmia compared with that provided by catheter-based techniques. This technology also has the potential to be used endoscopically, allowing for a truly minimally invasive procedure. Although these initial results are promising, clinical studies are needed to examine the effectiveness of these lesions in treating atrial fibrillation.

We thank Diane Toeniskoetter, Kathy Fore, and Dennis Gordon for their technical assistance.

\section{References}

1. Cameron A, Schwartz MJ, Kronmal RA, Kosinski AS. Prevalence and significance of atrial fibrillation in coronary artery disease (CASS registry). Am J Cardiol. 1988;61:714-7.

2. Reeder AS, Sastry BS, Thomas TP, et al. Atrial fibrillation and stroke in elderly hospitalized patients. Age Aging. 1986;15:89-92.

3. Wolf PA, Dawber TR, Thomas HE Jr, Kannel WB. Epidemiologic assessment of chronic atrial fibrillation and risk of stroke: the Framingham study. Neurology. 1979;28:973-7.

4. National Center for Health Statistics. Public use data tape documentation: 1995 National Ambulatory Medical Care Survey. Hyattsville, MD: National Center for Health Statistics, US Public Health Service; 1997.

5. Smith PK, Holman WL, Cox JL. Surgical treatment of supraventicular tachyarrhythmias. Surg Clin North Am. 1985;65:553-70.

6. Cox JL, Canavan TE, Schuessler RB, et al. The surgical treatment of atrial fibrillation: II. Intraoperative electrophysiologic mapping and description of the electrophysiologic basis of atrial flutter and atrial fibrillation. J Thorac Cardiovasc Surg. 1991;101:406-26.

7. Kannel WB, Wolf PA, Benjamin EJ, Levy D. Prevalence, incidence and prognosis and predisposing conditions for atrial fibrillation: population-based estimation. Am J Cardiol. 1998;82:2N-9N.

8. Jazayeri MR, Hempe SL, Sra JS, et al. Selective transcatheter ablation of the fast and slow pathways using radiofrequency energy in patients with atrioventricular nodal reentrant tachycardia. Circulation. 1992; 85:1318-28.

9. Jais P, Shah DC, Haissaguerre M, et al. Efficacy and safety of septal and left-atrial linear ablation for atrial fibrillation. Am J Cardiol. 1999;84:139R-46R.

10. Dagres N, Clague JR, Lottkamp H, Hindricks G, Breithardt G, Borggrefe M. Impact of radiofrequency catheter ablation of accessory pathways on the frequency of atrial fibrillation during long-term follow-up: high recurrence rate of atrial fibrillation in patients older than 50 years of age. Eur Heart J. 2001;22:423-7.

11. Hoenicke EM, Strange RG Jr, Patel H, Prophet GA, Damiano RJ Jr. Initial experience with epicardial radiofrequency ablation catheter in an ovine model: moving towards an endoscopic maze procedure. Surg Forum. 2000;81:79-82.

12. Xie B, Heald SC, Camm AJ, Rowland E, Ward DE. Radiofrequency catheter ablation of accessory atrioventricular pathways: primary failure and recurrence of conduction. Heart. 1997;77:363-8.

13. Rosenthal LS, Mahesh M, Beck TJ, et al. Predictors of fluoroscopy time and estimated radiation exposure during radiofrequency catheter ablation procedures. Am J Cardiol. 1998;82:451-8.

14. Yu WC, Hsu TL, Tai CT, et al. Acquired pulmonary vein stenosis after radiofrequency catheter ablation of paroxysmal atrial fibrillation. J Cardiovasc Electrophysiol. 2001;12:887-92.

15. Gerstenfeld EP, Guerra P, Sparks PB, Hattori K, Lesh MD. Outcome after radiofrequency catheter ablation of focal atrial fibrillation triggers. J Cardiovasc Electrophysiol. 2001;12:900-8. 